Light for controlled fusion energy: A perspective on laser-driven inertial fusion

This content has been downloaded from IOPscience. Please scroll down to see the full text. 2015 EPL 10945001

(http://iopscience.iop.org/0295-5075/109/4/45001)

View the table of contents for this issue, or go to the journal homepage for more

Download details:

IP Address: 151.100.44.29

This content was downloaded on 27/02/2015 at 15:03

Please note that terms and conditions apply. 


\title{
Light for controlled fusion energy: A perspective on laser-driven inertial fusion
}

\author{
Stefano Atzeni
}

Dipartimento SBAI, Università di Roma "La Sapienza" and CNISM - Via A. Scarpa, 14, 00161 Roma, Italy

received 17 February 2015; accepted in final form 17 February 2015

published online 27 February 2015

PACS 52.57.-z - Laser inertial confinement

PACS 52.57.Fg - Implosion symmetry and hydrodynamic instability (Rayleigh-Taylor,

Richtmyer-Meshkov, imprint, etc.)

\begin{abstract}
The status of laser-driven inertial confinement fusion research is briefly reviewed. The recent major achievement of fusion energy release exceeding the energy delivered by the laser to the fuel (Hurricane O. et al., Nature, 506 (2014) 343), and the efforts towards ignition demonstration using indirect-drive are discussed. Physics model reliability is addressed. The potentials of alternative schemes, in particular direct-drive shock ignition, are also illustrated.
\end{abstract}

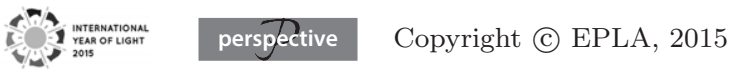

Introduction. - Very recently, for the first time, a laser-driven deuterium-tritium (DT) fuel attained a fuel gain larger than unity: the energy released by fusion reactions exceeded the energy the laser had delivered to the fuel [1]. The reaction involved is $D+T \rightarrow \alpha+n$, with a $Q$-value of $17.6 \mathrm{MeV}$. Prompted by this accomplishment and on the occasion of the International Year of Light, we present a brief overview and a personal perspective on laser-driven inertial confinement fusion (ICF) $[2,3]$.

Fusion energy research began in the early 1950s. The achievement of controlled fusion energy is still one of the grand challenges for the 21st century physics [4]. Two main alternative paths - magnetic confinement fusion (MCF) [5] and inertial confinement fusion [2] - are actively investigated. In both cases temperatures of the order of $10 \mathrm{keV}$ (in energy units) have to be achieved. In $\mathrm{MCF}$, a low-density DT plasma is confined with the help of intense magnetic fields. In ICF, instead, a small amount of DT (milligrams at most) is compressed by a powerful pulsed energy source to a density of hundreds of $\mathrm{g} / \mathrm{cm}^{3}[6]$. No means can be used to keep it confined: matter only remains compressed for the time allowed by its own inertia (hence the name), typically a fraction of a ns. This process is intrinsically pulsed. A future fusion reactor can be envisaged burning fuel elements (targets) at a rate of a few hertz. Each target would be irradiated by driver (laser) pulses of a few MJ, and should attain an energy gain (ratio of fusion energy to delivered driver energy) $G \geq 100$. More precisely, if we call $\eta_{\mathrm{d}}$ the electrical efficiency of the driver, the condition $G \eta_{\mathrm{d}} \geq 10$ should be satisfied [2,7]. The attainment of large $G$ requires the above-quoted compression as well as hot-spot ignition. Only a small portion of the fuel, a hot spot, should be heated at $5-10 \mathrm{keV}$ and initiate fusion burn. The energy deposited by the $\alpha$-particles should then self-heat the spot and drive a burn-wave propagating through the whole fuel.

Of course, a necessary major step towards energy production by laser fusion is the laboratory demonstration of ignition and onset of burn propagation. The following steps will concern the achievement of the gain required for net energy production and, eventually, the development of concepts and technology for economic energy production.

In this paper, we focus on ignition and on physics modelling. After brief remarks on laser fusion principles, we shall consider the results obtained by the US National Ignition Campaign. The goal is a brief discussion of the status of our understanding of the underlying physics, and then of the reliability of the models used by the ICF community. This in particular relates to the study of target concepts for power producing reactors. Such designs, indeed, rely on numerical simulations and involve rather large extrapolation of parameters with respect to present experiments. In the final part of the paper we discuss the potentials of the (laser-driven) shock ignition scheme, also taking advantage of the lessons learnt from the above ignition experiments. 
Laser fusion principles and requirements. - It may be surprising that ordered (and therefore expensive) laser light is used to (disorderly) heat matter and reach fusion conditions. In fact, as soon as the first $Q$-switched lasers were operated, scientists realized that unique features of pulsed lasers (in terms of deliverable power and intensity) made them ideal fusion drivers (see, e.g., [8,9], sect. II of ref. [3] and sect. 3.4 of ref. [2]).

In conventional laser fusion schemes [6] a millimetresized hollow spherical target, consisting of an inner layer of frozen DT fuel and an outer layer of a suitable supporting material is irradiated either by converging laser beams or laser-produced thermal radiation. The two approaches are referred to as direct-drive and indirect-drive, respectively (see fig. 1). Absorption of radiation causes heating, ionization and fast expansion of the outer layers. As a consequence of the outward expansion of the ablated materials, the remaining portion of the shell implodes. When it converges at the centre, its kinetic energy is converted into internal energy. A nearly isobaric configuration is generated, with a central ignition hot spot surrounded by denser and colder fuel.

In addition to efficient absorption of laser energy, and conversion to $\mathrm{X}$-rays in indirect-drive, this process requires i) efficient attainment of high implosion velocity $u$; ii) efficient compression of the bulk fuel; iii) symmetric implosion (to allow the generation of the central hot spot), and iv) limitation of the effects of Rayleigh-Taylor instability (RTI), which threatens shell integrity and can cause material mixing (for comprehensive treatments of RTI in ICF see sect. VI of ref. [3], sect. VI of [10], Chapt. 8 of [2]). The quality of compression, item ii) above, is usually gauged by the fuel isentrope parameter or adiabat $a=p / p_{\operatorname{deg}}>1$, with $p$ fuel pressure at peak implosion velocity and $p_{\text {deg }}$ the pressure of Fermi degenerate matter at the same density; the lower $a$, the more efficient the compression ${ }^{1}$.

According to an approximate criterion $[2,3,11]$, ignition occurs when the product $p_{\mathrm{h}} R_{\mathrm{h}}$ of hot-spot pressure and radius, exceeds a threshold value of $10-15$ Tbar $\cdot \mu \mathrm{m}$. The pressure of the hot spot, in turn, depends on the implosion velocity $u$, adiabat $a$ and ablation pressure driving the implosion, $p_{\mathrm{a}}$, scaling as $[12,13] p_{\mathrm{h}} \propto u^{3} a^{-9 / 10} p_{\mathrm{a}}^{2 / 5}$, which shows the crucial role of both implosion velocity and adiabat. Indeed, numerical simulations [14] show that the minimum laser energy for ignition $E_{\mathrm{L}-\mathrm{ig}}$ scales as

$$
E_{\mathrm{L}-\mathrm{ig}} \propto \eta^{-1} u^{-6} a^{-1.8} p_{\mathrm{a}}^{-0.8}
$$

(Note that this scaling is also recovered by a simple model [15].) However, while high velocity and low adiabat reduce the ignition energy, they increase the susceptibility to instabilities, by implying a thinner shell ${ }^{2}$ and lower degree of the ablative stabilization $[3,16]$ of RTI. In eq. (1) $\eta$

\footnotetext{
${ }^{1}$ We use the symbol $a$ instead of the more usual $\alpha$ to avoid confusion with $\alpha$-particles.

${ }^{2} \mathrm{~A}$ relevant parameter here is the in-flight aspect ratio, IFAR, ratio of the shell radius to the shell thickness during the implosion.
}

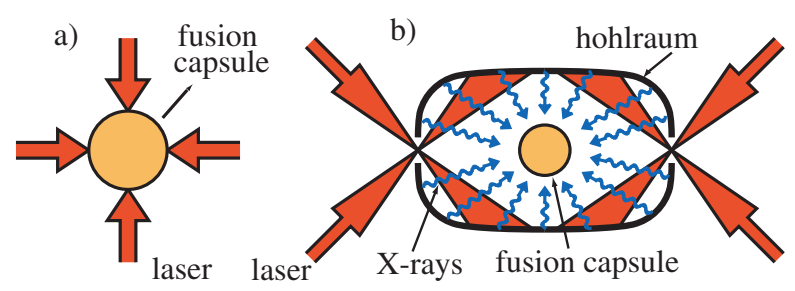

Fig. 1: (Colour on-line) Direct- (a) and indirect-drive (b) approaches to laser fusion.

is the laser-to-fuel coupling efficiency, i.e. the fraction of the laser beam energy delivered to the fuel.

In indirect-drive [3], the shell (often called capsule) is contained inside a cavity or hohlraum with high- $Z$ walls. The laser beams deliver their energy inside the cavity and generate thermal radiation (X-rays, with a temperature of about $300 \mathrm{eV}$ ), which, in turn, drives the implosion of the capsule [3]. Indirect-drive has a lower coupling efficiency $\eta$ than direct-drive, but sets less stringent requirements on the laser irradiation geometry and beam quality, and causes smaller RTI growth [3,10]. In the mid-1990s the experimental data on laser interaction and laser drive, hydrodynamic instabilities and matter compression by laser, integrated by massive numerical simulations, were deemed adequate to proceed to the design of a laser, the National Ignition Facility (NIF), and targets for ignition experiments using indirect-drive [3].

Ignition experiments at the NIF. - NIF [17] is a 192-beam Nd:glass laser, delivering pulses of up to $1.9 \mathrm{MJ}$ and peak power above $400 \mathrm{TW}$ on target. The laser light wavelength is $351 \mathrm{~nm}$, the third harmonic of the Nd:glass; VUV light is required to ensure nearly total absorption. A baseline target [18] for indirect-drive ignition experiments is shown in fig. 2. The fusion capsule, with outer radius of about $1.1 \mathrm{~mm}$, consists of an outer ablator layer of doped plastics and an inner layer of frozen DT (with mass $M_{\mathrm{DT}}=0.17 \mathrm{mg}$. The cylindrical hohlraum (made of $\mathrm{Au}$ or $\mathrm{U}$ ) is filled with moderate-density He gas, to limit the motion of the laser-heated hohlraum walls. The beams are delivered at different angles and have different powers, to ensure a nearly symmetric irradiation of the capsule. Irradiation uniformity also relies on the transfer of energy from different sets of beams through the non-linear plasma process of crossed-beam energy transfer, CBET [19]. Beam power is accurately shaped in time (see fig. 3) to produce a peak ablative driving pressure exceeding 100 Mbar only at the end of a sequence of steps of increasing pressure, in order to keep the adiabat small enough. This baseline target was designed to implode at $u>370 \mathrm{~km} / \mathrm{s}$ and adiabat $a=1.5$ [18], to achieve ignition with margins accounting for some performance degradation due to non-perfect symmetry and (minor) contamination of the fuel due to RTI. At implosion collapse, the central hot spot would have temperature exceeding $5 \mathrm{keV}$, density about $100 \mathrm{~g} / \mathrm{cm}^{3}$, pressure of $350 \mathrm{Gbar}$ and radius 


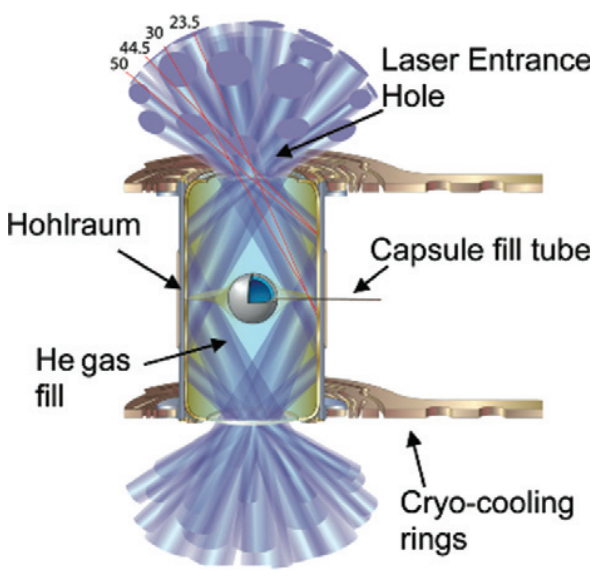

Fig. 2: (Colour on-line) Schematic of NIF ignition. Reprinted with permission from ref. [20], copyright 2011, AIP Publishing LLC.
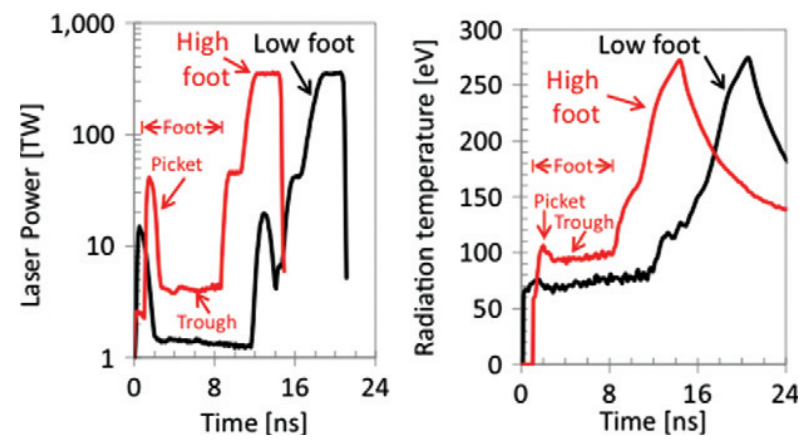

Fig. 3: (Colour on-line) NIF low-foot and high-foot laser pulses (left), and corresponding radiation temperature in the hohlraum (right). Reprinted with permission from ref. [48], copyright 2014 by the American Physical Society.

about 30 microns $(1 / 35$ the initial shell radius). The cold fuel would be compressed at about $1000 \mathrm{~g} / \mathrm{cm}^{3}$, with a confinement parameter $\langle\rho R\rangle=\int \rho \mathrm{d} r=1.5 \mathrm{~g} / \mathrm{cm}^{2}$.

NIF was commissioned in 2009, and soon achieved and later even exceeded design specifications [11]. A National Ignition Campaign (NIC [18,20,21]) was conducted from 2009 to 2012 (see ref. [11] for a comprehensive review). NIC implosions achieved values of fuel compression and confinement parameter close to the goal (e.g., $\langle\rho R\rangle=$ $\left.1.3 \mathrm{~g} / \mathrm{cm}^{2}\right)$, but the hot-spot pressure was a factor $2.5-3$ lower than expected and ignition was not achieved. The maximum neutron yield $Y_{\mathrm{n}}$ was about $10^{15}$. Detailed comparison between simulation and experiments was possible thanks to diagnostics [20] with unprecedented space- and time-resolution. These include, e.g. hard X-ray radiography [22], X-ray imaging [23], neutron imaging [24]. For instance, it was possible to obtain $2 \mathrm{D}$ images of the imploding shells [22], as well as X-ray and neutron images of the hot spot. Measurements, reviewed in detail in ref. [11] show departures from predictions in a few areas: i) laser light backscattering losses (about 15\%), due to laser plasma interactions (LPIs) are larger than expected (for a recent reviews on LPIs in indirect-drive see [25]); ii) the implosion velocity was about $10 \%$ smaller than computed for a given absorbed laser energy [26]; iii) the hot-spot internal energy was smaller than expected at a given implosion velocity [27]; this was attributed to asymmetries causing incomplete conversion of kinetic energy into internal energy at the end of the implosion [28]; iv) when the implosion velocity exceeded some threshold value $(300-340 \mathrm{~km} / \mathrm{s}$, depending on target and pulse details), the $Y O C$ (yield over clean, ratio of the actual fusion yield to that expected from 1D simulations) dropped from about $20 \%$ to below $5 \%$ (probably due to material mix caused by RTI [29]).

The NIC outcome suggested to put emphasis on understanding the above individual issues i)-iv), by performing focused experiments, before attempting again an integrated approach to ignition [30,31]. A few specific experiments which have already shed light on key issues will be mentioned later when discussing model reliability. Here we consider the record yield experiments reported in refs. [1,32-34] and quoted at the beginning of this paper. Such experiments differed from the NIC implosions for the laser power pulse shape, which was changed by increasing the power of the foot, reducing the number of shock-driving steps, and shortening the pulse itself (see fig. 3). This new high-foot pulse produces a stronger first shock and increases the fuel adiabat to $a \simeq 2.5$. This reduces compressibility, increasing shell thickness (proportional to $a^{3 / 5}$ ) and reducing RTI growth rate. Of course, also the final compression is reduced, with the beneficial effect of reduced sensitivity to asymmetry due to smaller convergence, and the negative effect of a smaller $\langle\rho R\rangle \leq 0.8 \mathrm{~g} / \mathrm{cm}^{2}$. The neutron yield $Y_{\mathrm{n}}$ reached $6 \times 10^{15}$ in the experiments of ref. [1,33] and $9.3 \times 10^{15}(26 \mathrm{~kJ}$ of fusion energy) in subsequent experiments [34], exceeding by a factor 10 the yield of low-foot experiments. The yield was multiplied by self-heating by a factor $\mathcal{M}_{y} \simeq 2$, and the fuel energy gain, ratio of fusion energy to fuel energy, was also about 2. Even more important, reaction yields from simulations using the experimental temperature drive were found in good agreement with the experimental ones, so that $Y O C=50-70 \%$.

Ignition metrics. - How far are these experiments from the demonstration of ignition and burn propagation? To address this question we have to introduce appropriate metrics, and analyse their dependence on implosion parameters. The highest fusion yield so far, $26 \mathrm{~kJ}$, is 40 times below the $1 \mathrm{MJ}$ defining ignition in the NIC [11]. However, ignition is a threshold process, and the yield is not the best metrics of performance. Changing the implosion velocity, or decreasing fuel reactivity by a few percent can lead to order-of-magnitude differences in yield, as shown by many different studies referring to different target concepts (see, e.g., refs. [35-40]). A more suitable quantity is the factor $\chi$ obtained from a generalization [41] of the Lawson criterion used in magnetic confinement fusion [5]. It is given by the 


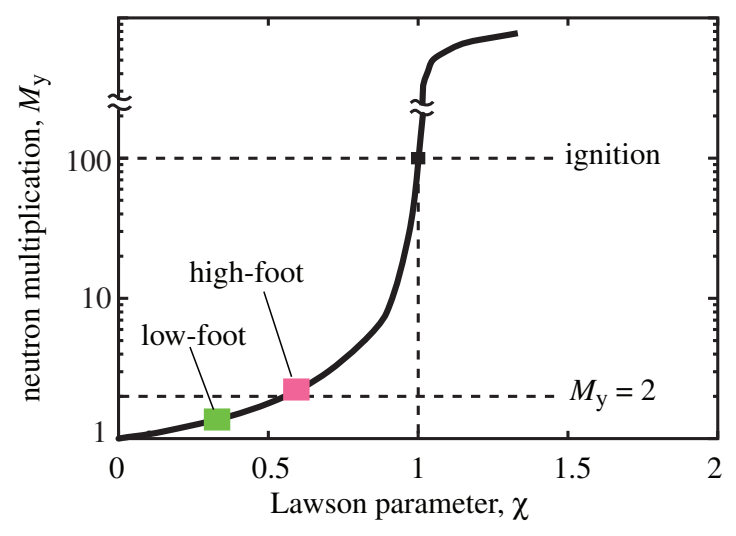

Fig. 4: (Colour on-line) Yield multiplication due to $\alpha$ selfheating vs. Lawson parameter $\chi$. The highest values of $\chi$ obtained in low-foot [11] and high-foot experiments [34] are also shown.

product $p \tau$ of the pressure of the imploded fuel prior to eventual fusion self-heating times the confinement time, normalized to the value $(p \tau)_{\text {ig }}$ required for ignition. The use of $\chi$ is particularly useful because i) yield multiplication $\mathcal{M}_{y}$ is a function of $\chi, \mathcal{M}_{y}=\mathcal{M}_{y}(\chi)$; ii) $\chi$ can be determined from two measurable quantities [37], namely neutron yield $Y_{\mathrm{n}}$ and $\langle\rho R\rangle$, in turn obtained from the ratio of downscattered neutrons to non-scattered neutrons [38]. Formally, $\chi \propto\langle\rho R\rangle^{0.61}\left(Y_{\mathrm{n}} / M_{\mathrm{DT}}\right)^{0.24}\left[\mathcal{M}_{y}(\chi)\right]^{-1 / 3}$. Highfoot experiments achieved $\chi$ up to about 0.6 , vs. $\chi \leq 0.3$ in low-foot experiments. The behaviour of $\mathcal{M}_{y}$ vs. $\chi$ is illustrated in fig. 4. It shows that increasing $\chi$ by $60-70 \%$ would lead to ignition and a 50-100 times larger yield.

How can this increase of $\chi$ be achieved? An insight is provided by the Ignition Threshold Factor [38,39], ITF, a metric closely related to $\chi[11]$, but expressed in terms of different parameters,

$$
\begin{aligned}
\chi \approx I T F^{\delta} & =\left(I T F_{1 \mathrm{D}} f_{\mathrm{h}} f_{\mathrm{mix}} f_{\mathrm{RKE}}\right)^{\delta}, \\
I T F_{1 \mathrm{D}} & \propto M_{\mathrm{DT}} u^{8} a^{-2.6},
\end{aligned}
$$

where $\delta=0.38-0.45$, and $f_{\mathrm{h}}, f_{\mathrm{mix}}$, and $f_{\mathrm{RKE}}$ are factors accounting for deviations from 1D behaviour, namely, hotspot deformation, material mix in the hot spot, and residual kinetic energy, respectively (see refs. [11] and [42] for their expressions). Equation (3) evidences again the critical role of both implosion velocity and adiabat, as well as the need for the generation of a clean, symmetric hot spot. In the high-foot implosions [1,32-34] the $f$ 's were not far from unity, but the price to pay was a higher $a$.

Current investigations aim at increasing the coupling efficiency $\eta$ (note that $\left.M_{\mathrm{DT}} u^{8}=\left(M_{\mathrm{DT}} u^{2}\right) u^{6}=2 \eta E_{\mathrm{L}} u^{6}\right)$, and slightly increasing the implosion velocity at the same time controlling symmetry and limiting RTI development. Options already successfully tested in specific experiments include reducing hohlraum gas fill density and using highdensity carbon ablators [43], and changing the hohlraum shape [44]. Better RTI control could be achieved by implementing an adiabat-shaping technique, similar to that used in direct-drive [45]. Adiabat shaping consists in space-profiling the adiabat in the shell, keeping it relatively large in the outer region of the shell (so that RTI growth is reduced), and low in the inner fuel region in order to allow for efficient compression. It can be achieved by using properly time-shaped laser pulses. It has of course to be proved that all these separate improvements can be achieved simultaneously at ignition scale without degrading implosion symmetry.

Modelling and simulation. Lessons from the NIF. - According to a December 2012 US DoE report to the Congress on the NIC campaign [30] "experimental data demonstrate that the physics underlying target implosions are not predicted accurately by the simulation codes that were used to design ignition targets". Does this apply to ICF in general? Which models should be improved? How can one trust computer simulations of innovative design concepts?

Discrepancies between predictions and measurements mainly concern two aspects. The first one is hohlraum physics when the hohlraum is filled with relatively highdensity gas, and LPIs play a key role. Experiments have however proved that when LPIs are less important, as in hohlraums with very low gas fill density and/or when pulses are shorter so that plasma formation and motion is limited, state-of-the-art radiation-hydrodynamics models [46] are predictive [47]. A second area of difficulty concerns mix due to non-linear RTI evolution (linear RTI growth rates are instead accurately predicted [48]). However, a very recent paper indicates that when geometric details of the capsule, including fill tube and supporting tent, are taken into account, code predictivity improves substantially [49]. NIF exploding pusher experiments also show that both implosion hydrodynamics and nuclear modelling are very accurate [50]. It is also worth mentioning that while fluid models are appropriate in most cases, recent experimental results [51] suggest that kinetic effects could play some role when the first shock crosses the inner vapour or in hohlraum filled with very low density gases.

It then turns out that state-of-the-art radiationhydrodynamics models seem adequate to simulate targets under conditions where LPIs are not important and RTI not yet in fully non-linear regimes; efforts should be made to design targets operating under such conditions. Target designs should in any case include large safety margins, to account for unavoidable uncertainties.

A promising alternative: direct-drive, with shock ignition. - Indirect-drive was chosen as the primary candidate for ignition about two decades ago [3]. At that time, the disadvantage of indirect-drive with respect to direct-drive (less efficient energy coupling to the fuel due to the intermediate conversion to X-rays) was deemed overbalanced by the less stringent laser beam requirements to achieve uniform drive and the lower RTI growth rate of X-ray-driven ablation [3]. Since then, however, great progress has been made in laser technology and, 
particularly, in beam-smoothing techniques [52,53]. Furthermore, the introduction of adiabat-shaping [45] makes low-to-moderate adiabat, direct-drive implosions conceivable. Successful experiments on scaled-down ignition targets $[54,55]$ have been performed at the OMEGA laser facility [56]. Also, polar direct-drive designs [57,58] have been produced for direct-drive implosions using the beams of lasers originally designed for indirect drive, such as NIF and Laser MegaJoule (LMJ [59], currently under construction). In addition, we notice that direct-drive schemes do not involve the very complex hohlraum physics (apparently not yet fully understood, as seen in the previous section), and employ much simpler targets.

However, issues still remain concerning irradiation uniformity at the beginning of laser pulse, potential deleterious effects of CBET (due to beam overlapping) [60], and RTI control at low adiabat. Such issues, which are essentially related to the need of large implosion velocity and large in-flight aspect ratio are somewhat reduced in directdrive concepts employing advanced ignition schemes. The above-mentioned progress in direct-drive therefore supports advanced schemes and, in particular, shock ignition.

Advanced ignition schemes (see ref. [61] for a series of updated reviews) are two-step processes. The fuel is first imploded as in conventional ICF, but at lower implosion velocity. The ignition hot spot is created in the precompressed fuel by an additional pulse [62].

A first advanced scheme is fast ignition, which foresees hot-spot heating by relativistic electrons [62] or energetic ions [63,64], in turn accelerated by strongly focused ultraintense laser pulses. The issue here is the efficient generation of focused particle beams and/or their transport through the compressed plasmas [61,65-67]. Unfortunately, present and near-future facilities do not allow full-scale tests of the scheme, which is hardly scalable to smaller dimensions.

In shock ignition [68], instead, the hot spot is generated with the help of a strong spherically convergent shock wave driven by a spike pulse launched towards the end of the implosion by the same laser used for compression (see fig. 5). A small-scale proof-of-principle experiment was conducted soon after the first proposal [69]. Subsequent studies indicated that shock ignition has potential for high gain at MJ laser energy [40,68,70-74] (see fig. 6) and could be tested using polar-direct-drive schemes at NIF $[75,76]$ (or at the Laser MegaJoule, LMJ [59]). In addition, most of its aspects can be studied in scaled-down experiments. Potential advantages of shock ignition vs. conventional ignition (higher gain, reduced susceptiblity to RTI) follow from the reduced implosion velocity [68].

A distinctive feature of shock ignition is that two parameters, namely implosion velocity and spike power can be adjusted to achieve ignition; of course, the lower the velocity, the higher the spike power. Since RTI risks increase with velocity, while LPI risks with spike intensity, this introduces some degree of flexibility and allows for potential risk reduction. a)

b)
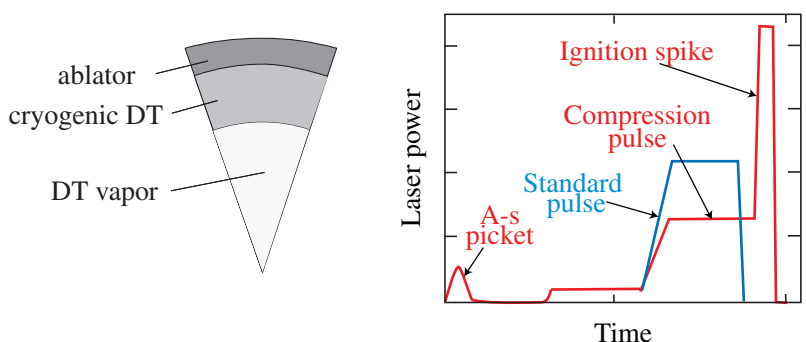

Fig. 5: (Colour on-line) (a) Target for direct-drive laser fusion; (b) laser pulses for conventional direct-drive (blue) and for shock ignition (red). Reprinted with permission from ref. [40], copyright 2015 by the Institute of Physics.

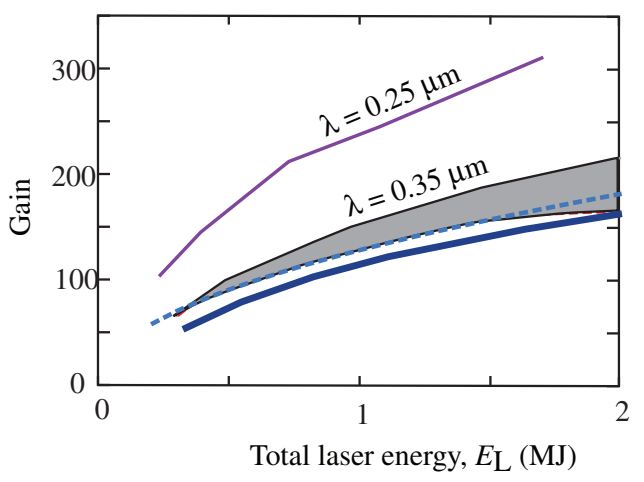

Fig. 6: (Colour on-line) Gain curves for shock ignition. The upper curve refers to targets irradiated by laser light with wavelength $\lambda=0.25 \mu \mathrm{m}$ [72]; all others and the grey area refer to targets driven by $\lambda=0.35 \mu \mathrm{m}$ light $[40,71,73,74]$.

Of course, shock ignition has its own specific issues. According to the studies reviewed in refs. [77] and [78], these are i) efficient absorption of the laser spike (with intensity about $\times 10^{16} \mathrm{~W} / \mathrm{cm}^{2}$, causing intense LPIs) and generation of a pressure of the order of 300-400 Mbar; ii) limiting deleterious effects of the LPIs expected to occur during spike interaction (light backscattering and production of hot electrons, in particular); iii) sensitivity to laser pointing/target positioning errors, due to the high convergence ratio [79]. Recent results, however, have provided at least partial answers to the above issues. An experiment at OMEGA demonstrated the generation of pressure exceeding $300 \mathrm{Mbar}$ [80]; pressure was even strengthened by laser-generated suprathermal electrons, supporting recent theoretical and computational suggestions [81]. Notice that such hot electrons do not preheat the bulk fuel, hindering compression, because they are produced at the later stages of the implosion, when the shell thickness exceeds the suprathermal eletron range [82]. Finally, models and simulations show that the robustness of shock ignition targets to pointing/positioning errors can be greatly increased by increasing the target 1D ignition safety margin [40] (measured by $I T F^{*}$, a variant $[37,75]$ of the $I T F_{1 \mathrm{D}}$, suited to shock ignition). 


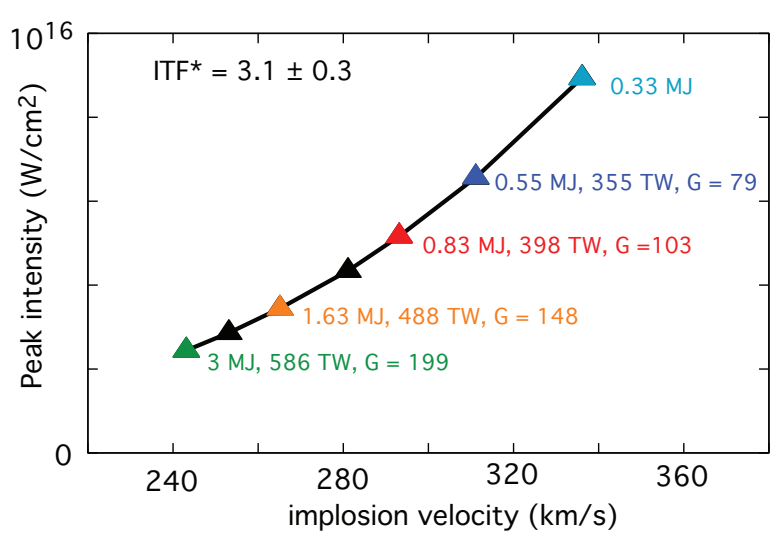

Fig. 7: (Colour on-line) Spike intensity vs. implosion velocity, for a family of geometrically similar shock ignition targets, with approximately the same value of the ignition threshold factor. Laser energy and power are also indicated along the curve. For details, see ref. [40].

Figure 6 summarizes gain curves obtained by various authors. Typical values of implosion velocity, spike intensity and power of a (preliminary) shock ignition design are shown in fig. 7 for a family of geometrically similar targets with approximately the same value of $I T F^{*}$. It turns out that shock ignition could be tested at full scale with a laser with pulse power and energy available on NIF. For a detailed target design, discussion of the relevant polardirect-drive scheme, required NIF beam modifications and safety margins, see ref. [75].

Conclusions. - I have presented a brief overview of laser fusion research, focusing first on recent NIF achievements in the indirect-drive approach. The enormous amount of accurate data acquired in the past few years has led to a significant improvement in our understanding of ICF physics, and has shown in which areas models are fully adequate and in which other areas (e.g. LPI and non-linear RTI, radiation-hydrodynamics in complex geometries) further modelling progress is needed. This also suggests designing targets operating in more predictable regimes. Indirect-drive performance improvement has been achieved just following this way. Future experiments will show whether this will allow achieving ignition in indirect-drive on the NIF. Important progress has also been achieved in direct-drive, which has potentials for higher energy gain and employs much simpler targets. Experiments so far have been conducted at an intermediate-scale facility (OMEGA laser [56], with laser pulses of about $30 \mathrm{~kJ}$ ), and tests at larger scale are required. While direct-drive does not involve the extremely complex hohlraum physics, LPIs (and in particular CBET) could still play some role, and RTI still sets stringent limitations. This leads to the interest in shock ignition, where the implosion velocity, and hence RTI risks, are reduced. Results on shock ignition indicate, however, that the concept is still in its infancy, and full-scale integrated experiments are required. It is worth remarking that full-scale investigation of both conventional directdrive and shock ignition is in principle possible at NIF and LMJ.

The achievement of fusion energy in excess of the fuel energy, as well as the demonstration of significant hot-spot self-heating in high-foot experiments are landmarks in inertial fusion research. A progress of a factor about 2 in the Lawson parameter $\chi$ would lead to ignition, $\mathcal{M}_{y}>100$ and target gain $G>1$. While the path to fusion energy production will still be long [83] (requiring $G \approx 100$, laser efficiency about $10 \%$ and operation at a few hertz), ignition in the laboratory will demonstrate the soundness and scientific feasibility of the scheme. Finally, it is also worth recalling that lasers such as NIF and MJ also offer unique opportunities to high-energy density science [17], in particular to the emerging field of laboratory astrophysics $[84,85]$.

I acknowledge the efforts and achievements of the large ICF community. However, the responsibility for any comments and remarks is mine only. This work was partially supported by the Italian project PRIN 2012AY5LEL. I also acknowledge LLNL-NIC invitation (and related funding) to the 2011 NIC Study Group, as well as NNSA support for my participation in the Science of Fusion Ignition on NIF Workshop, May 22-24, 2012. I thank A. Marocchino and A. Schiavi for their collaboration in shock ignition studies.

\section{REFERENCES}

[1] Hurricane O. et al., Nature, 506 (2014) 343.

[2] Atzeni S. and Meyer-Ter-Vehn J., The Physics of Inertial Fusion (Oxford University Press) 2004.

[3] Lindl J. D., Phys. Plasmas, 2 (1995) 3933.

[4] Ginzburg V. L., Usp. Fiz. Nauk, 169 (1999) 419.

[5] Freiberg J. P., Fusion Energy and Plasma Physics (Cambridge University Press) 2007.

[6] Nuckolls J. et al., Nature, 239 (1972) 139.

[7] Hogan J. W. (Editors), Energy from Inertial Fusion (International Atomic Energy Agency, Vienna) 1995.

[8] Nuckolls J. H., Early steps towards inertial fusion energy 1952-1962, report UCRL-JC-131075 Rev 1 (Lawrence Livermore National Laboratory, Livermore, Cal., USA) 1999.

[9] Kidder R. E., SPIE Proc., 3343 (1998) 10.

[10] Lindl J. D. et al., Phys. Plasmas, 11 (2004) 339.

[11] Lindl J. D. et al., Phys. Plasmas, 21 (2014) 020501.

[12] Meyer-Ter-Vehn J. and Schalk C., Z. Naturforsch., 37a (1982) 955.

[13] Kemp A., Meyer-ter-Vehn J. and Atzeni S., Phys. Rev. Lett., 86 (2001) 3336.

[14] Herrmann M. C., Tabak M. and Lindl J. D., Nucl. Fusion, 41 (2001) 99.

[15] Atzeni S. and Meyer-Ter-Vehn J., Nucl. Fusion, 41 (2001) 465.

[16] Betti R. et al., Phys. Plasmas, 5 (1998) 1446. 
[17] Miller G. H., Moses E. I. and Wuest C. R., Nucl. Fusion, 44 (2004) S228.

[18] HaAn S. W. et al., Phys. Plasmas, 18 (2011) 051001.

[19] Michel P. A. et al., Phys. Plasmas, 17 (2010) 056305.

[20] Edwards M. J. et al., Phys. Plasmas, 18 (2011) 051003.

[21] Landen O. L. et al., Phys. Plasmas, 18 (2011) 051002.

[22] Rygg J. R. et al., Phys. Rev. Lett., 112 (2014) 195001.

[23] Bell P. M. et al., Rev. Sci. Instrum., 81 (2010) 10E540.

[24] Merrill F. E. et al., Rev. Sci. Instrum., 83 (2012) $10 \mathrm{D} 317$.

[25] Kirkwwod R. K. et al., Plasma Phys. Control. Fusion, 55 (2013) 103001.

[26] Hicks D. G. et al., Phys. Plasmas, 19 (2012) 122702.

[27] Cerjan C., Springer P. T. and Sepke S. M., Phys. Plasmas, 20 (2013) 056319.

[28] Sсотт R. H. H. et al., Phys. Rev. Lett., 110 (2013) 075001.

[29] Regan S. P. et al., Phys. Rev. Lett., 111 (2013) 045001.

[30] National Nuclear Security Administration's Path Forward to Achieving Ignition in the Inertial Confinement Fusion Program (United States Department of Energy, Washington, DC, USA) December 2012.

[31] Science of Fusion Ignition on NIF Workshop, May 22-24, 2012 (Lawrence Livermore National Laboratory, Livermore, Cal., USA) 2012, LLNL-TR-570412.

[32] Park H.-S. et al., Phys. Rev. Lett., 112 (2014) 055001.

[33] Hurricane O. et al., Phys. Plasmas, 21 (2014) 056314.

[34] Hurricane O. et al., Progress towards Ignition on the National Ignition Facility (Lawrence Livermore National Laboratory, Livermore, Cal., USA) November 10, 2014, LLNL-PRES-665679.

[35] Atzeni S., Europhys. Lett., 7 (1990) 639.

[36] Kishony R. and Shvarts D., Phys. Plasmas, 8 (2001) 4295.

[37] Chang P. Y. et al., Phys. Rev. Lett., 104 (2010) 135002.

[38] Spears B. K. et al., Phys. Plasmas, 19 (2012) 056316.

[39] Clark D. S., Haan S. and Salmonson J. D., Phys. Plasmas, 15 (2008) 056305.

[40] Atzeni S., Schiavi A. and Marocchino A., Plasma Phys. Control. Fusion, 57 (2015) 014022.

[41] Betti R. et al., Phys. Plasmas, 17 (2010) 058102.

[42] Kritcher A. L. et al., Phys. Plasmas, 21 (2014) 042708.

[43] Mac Kinnon A. J. et al., Phys. Plasmas, 21 (2014) 056318 .

[44] Amendt P. et al., Phys. Plasmas, 21 (2014) 112703.

[45] Anderson K. S. and Betti R., Phys. Plasmas, 11 (2004) 5.

[46] Rosen M. D. et al., High Energy Density Phys., 7 (2011) 180.

[47] Mac Laren S. A. et al., Phys. Rev. Lett., 112 (2014) 105103.

[48] Casey D. T. et al., Phys. Rev. E, 90 (2014) 011102(R).

[49] Clark D. S. et al., Phys. Plasmas, 22 (2015) 022703.

[50] Le Pape S. et al., Phys. Rev. Lett., 112 (2014) 225002.

[51] Rosenberg M. J. et al., Phys. Rev. Lett., 112 (2014) 185001 .
[52] Lehmberg R. H. and Obenschain S. P., Opt. Commun., 46 (1983) 27.

[53] Skupsky S. et al., J. Appl. Phys., 66 (1989) 3456.

[54] McCrory R. L. et al., Nucl. Fusion, 53 (2013) 113021.

[55] Goncharov V. N. et al., Phys. Plasmas, 21 (2014) 056315.

[56] Boehly T. R. et al., Opt. Commun., 133 (1997) 495.

[57] Skupsky S. et al., Phys. Plasmas, 11 (2004) 2763.

[58] Collins T. J. B. et al., Phys. Plasmas, 19 (2012) 056308.

[59] Ebrardt J. and Chaput J. M., J. Phys.: Conf. Ser., 244 (2010) 032017.

[60] Igumenshchev I. V. et al., Phys. Plasmas, 19 (2012) 056314.

[61] Special Issue: Collection of Special Topic papers on Inertial Confinement Fusion, Nucl. Fusion, 54, issue No. 5 (2014).

[62] Taвak M. et al., Phys. Plasmas, 1 (1994) 1626.

[63] Roth M. et al., Phys. Rev. Lett., 86 (2001) 436.

[64] Atzeni S., Temporal M. and Honrubia J. J., Nucl. Fusion, 42 (2002) L1.

[65] Atzeni S. and TABak M., Plasma Phys. Control. Fusion, 47 (2005) B769.

[66] Bellei C. et al., Phys. Plasmas, 19 (2012) 072711.

[67] AtZeni S., Inertial Fusion with Advanced Ignition Schemes: Fast Ignition and Shock Ignition, in Laser Plasma Interactions and Applications, edited by MC Kenna P. et al. (Springer) 2013, p. 243.

[68] Betti R. et al., Phys. Rev. Lett., 98 (2007) 155001.

[69] Theobald W. et al., Phys. Plasmas, 15 (2008) 056306.

[70] Ribeyre X. et al., Plasma Phys. Control. Fusion, 51 (2009) 015013.

[71] Perkins L. J., Betti R., LaFortune K. N. and Williams W. H., Phys. Rev. Lett., 103 (2009) 045004.

[72] Sсhmitt A. J. et al., Phys. Plasmas, 17 (2010) 042701.

[73] Atzeni S., Marocchino A., Schiavi A. and Schurtz G., New J. Phys., 15 (2013) 045004.

[74] Lafon M., Ribeyre X. and Schurtz G., Phys. Plasmas, 17 (2010) 052704.

[75] Anderson K. S. et al., Phys. Plasmas, 20 (2013) 056312.

[76] Perkins L. J. et al., in Fusion Energy 2010 (Proceedings of the 23rd International Conference, Daejon, 2010) (IAEA, Vienna) 2010, CD-ROM file IFE/P6-13.

[77] Atzeni S. et al., Nucl. Fusion, 54 (2014) 054008.

[78] Batani D. et al., Nucl. Fusion, 54 (2014) 054009.

[79] Atzeni S., Schiavi A. and Marocchino A., Plasma Phys. Control. Fusion, 53 (2011) 035010.

[80] Nora R., Phys. Rev. Lett., 114 (2015) 045001.

[81] Gus'kov S. et al., Phys. Rev. Lett., 109 (2012) 255004.

[82] Betti R. et al., J. Phys.: Conf. Ser., 112 (2008) 022024.

[83] Board on Physics and Astronomy, National ReSEARCh Council, An Assessment of the Prospects for Inertial Fusion Energy (National Academies Press, Washington, DC, USA) 2013.

[84] Remington B. A., Drake R. P. and Ryutov D. D., Rev. Mod. Phys., 78 (2006) 755.

[85] Bulanov S. V. et al., Eur. Phys. J. D, 55 (2009) 483. 